\title{
VISUALIZATION OF CAVITATING MICRO JETS
}

\author{
Róbert OLŠIAK, Branislav KNÍŽAT, Marek MLKVIK•
}

\begin{abstract}
The paper deals with one experimental set up integrated for research of the cavitating micro flows, which is incipient behind the micro channel or micro discharge nozzle outlet port. Experimental system is integrated from three major systems: hydraulic circuit with installed discharge nozzle (or micro channel), subsystem for data acquisition and data processing (DAQ system) and vision system compound of high speed video camera and pulse light source with highfrequency repetition. First few results of experiments (parameters such as inlet pressure, downstream pressure were changed) is also discussed.;
\end{abstract}

\section{INTRODUCTION}

Most publications, where presenting results of their work are most often focused on the fluid flow in channels of very small scales, where the cross section of the channel is of a non-circular shape (rectangular, square, trapezoidal....). Hydraulic characteristics of the tested micro fluidics devices are strictly depending on used manufacturing technology (most often technologies also used in manufacturing of semiconductors, MEMS components etc.: lithography, microlithography, etching wet process, dry process [6], micromachining, etc.). In the next part of the paper there are described experiments focused on the analysis of cavitation beginning and continuance by a high speed flow through the jet of a microscopic scale. For experimental research of cavitation in hydraulics devices with micro scale is used experimental test ring, which enabling investigation of cavitation (bubble, cloud) by visualisation method in parallel with measurement of hydraulic parameters and cavitation attendants (e.g. acoustic emission, noise, luminescence, etc). Results from realised experiments are analysed by advanced digital signal processing methods (vision data and DAQ data too).

\section{TEST RIg LAYOUT}

Experimental system is integrated from three major systems: hydraulic circuit with installed discharge nozzle (or micro channel), system for data acquisition and data processing (DAQ system) and vision system compound of high speed video camera and pulse light source with high-frequency repetition. All of the systems are assembled from great number of subsystems and separate component, however, test rig is relatively complicated technical equipment. The block diagram introducing of the test rig is in the Figure 1. In the following are described the subsystems and components (with briefly definition of his functions and parameters). Functions and parameters of assembled

\footnotetext{
- Róbert Olšiak (robert.olsiak@stuba.sk), Branislav Knížat (branislav.knizat@stuba.sk), Marek Mlkvik (marek.mlkvik@stuba.sk)
}

This is an Open Access article distributed under the terms of the Creative Commons Attribution License 2.0, which permits unrestricted use, distribution, and reproduction in any medium, provided the original work is properly cited. 
subsystem and components having fundamental influence on application field and quality and quantity of the experimental data, they will be discussed in next chapters.

Hydraulic circuit (Figure 1. sign. 1A) includes: the model of the investigated micro devices (micro orifice, channel, etc.) placed on the carrier unit made from a transparent material. Modular design of this component is enabling easy modify and change construction parts.

Hydraulic subsystem (sign. 1B) consisting of a source of hydraulic fluid on the inlet port of the channel (hydraulic pump unit) and vacuum recipient on the outlet port. For more information about hydraulic circuit see previous papers [7].
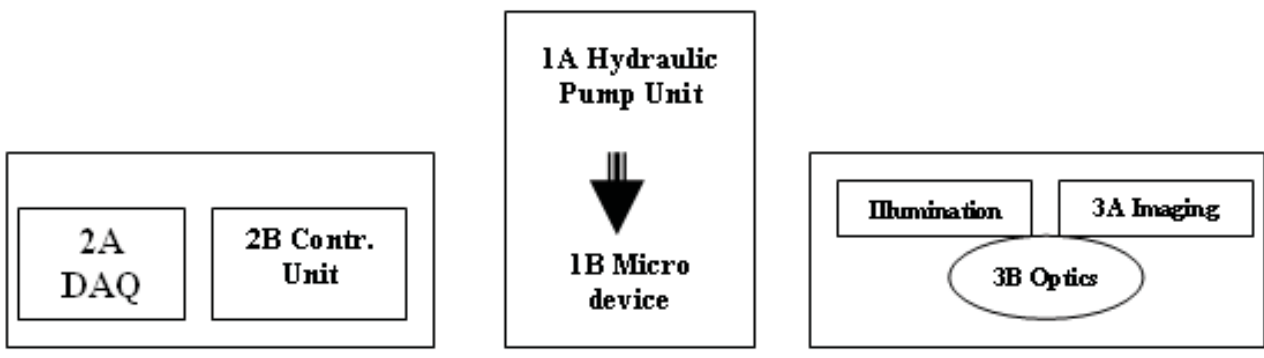

Figure 1: Scheme of the test rig

DAQ \& Control Unit (Figure 1. sign. 2A, and 2B), where is used for measurement of physical parameters, transmission, archiving and manipulation with non-visual data. System is built-in as a Power Workstation (inside INTEL Xeon processor) with add on PCI, resp. PCI-e DAQ and Control card. For visual observation cavitation (bubble or cloud) the vision system is necessary. Vision system, in presented application, is built-up from three subsystems (Figure 1. sign. 3A, 3B and $3 \mathrm{C}$ ). The first: digital imaging subsystem 3A, is intended for video data capturing and transferring to DAQ system. The second: optics subsystem $3 \mathrm{~B}$ is intended for transport of the optical data (optical image) to vision subsystem. The third: illumination subsystem 3C, is intended for illuminate of examined phenomenon.

\section{VISION SYSTEM DESCRIPTION}

Visualization is frequently used technique for cavitation phenomenon observation in macro scale hydraulic devices [1], [2], but in this time in micro scale hydraulic devices too. An vision system should create sufficient image quality to allow one to extract desired information about the phenomenon from the image. Note that what may be adequate image quality for one application may prove inadequate in another. There are a variety of factors that contribute to the overall image quality, including resolution, image contrast, depth of field, perspective errors, and geometric errors (distortion). In Figure 2 are briefly illustrated fundamental parameters of vision system.

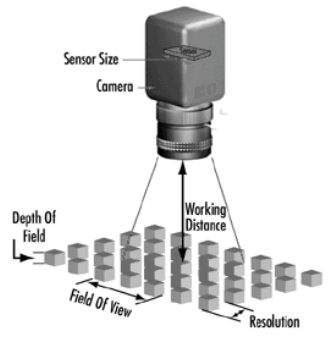

Figure 2: Imaging system parameters 
The setup with three cameras has been used for further visualization analysis: - $1^{\text {st }}$ configuration based on commercial digital camera OLYMPUS 7070 WZ, capture stationary images or color video stored in XD memory card. Only local display, export video data is necessary.

- $2^{\text {nd }}$ configuration based on laboratory HiRes analog video camera SONY ExWave (CCD sensor inside). Analog video signal is attached to DAQ Workstation via High Performance PCI Frame Grabber National Instruments.

- $3^{\text {th }}$ configuration based on High Speed digital video camera Redlake Y3 (CMOS sensor inside). Video data are stored in embedded video memory, and consequently transferred in DAQ Workstation via USB or Giga Ethernet link.

All digital imaging systems is fitted with optical equipment. Camera OLYMPUS 7070 WZ is used with integrated lenses OLYMPUS ED WIDE 5.7-22.9, f 1:208-4.8. HiRes analog video camera SONY ExWave is used with microscopic objectives build in laboratory microscope LEICA. High Speed digital video camera Redlake Y3 is used with NIKON $50 \mathrm{f}$ 0.98 objective or MITUTOYO telecentric objectives (constant magnification). They are easily integrated into C-Mount imaging systems with our Mitutoyo C-Mount camera tube, Figure 3. What are preference of latest configuration? Long Working Distances allow for integration into Vision Measurement System (much greater than laboratory microscope). Compact Design and long parfocal length simplifies mounting (needed for test rig modification). Mitutoyo telecentric objectives are ideal for high-resolution, high power vision measurement and optical gauging systems (e.g. small or moving objects).

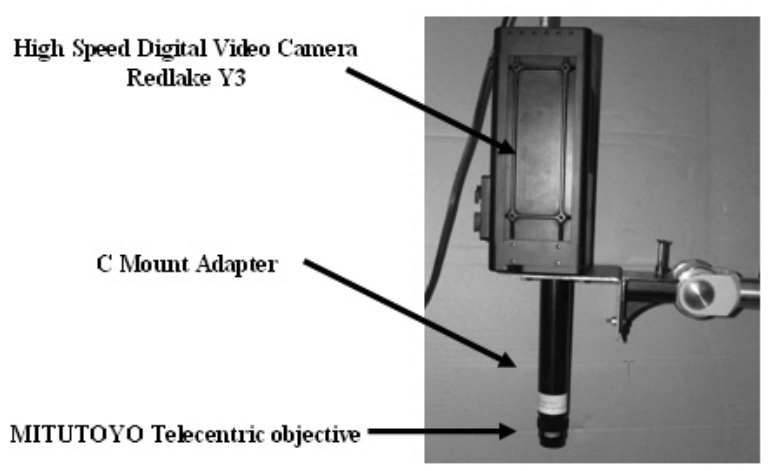

Figure 3: Imaging system

Correct illumination is critical to an image system and improper illumination can cause a variety of image problems. Blooming or hot spots, for example, can hide important image information, as can shadowing. In addition, shadowing can also cause false edge calculations when measuring, resulting in inaccurate measurements. Poor illumination can also result in a low signal-to-noise ratio. Non-uniform lighting, in particular, can harm signal-to-noise ratios and make tasks such as thresh holding more difficult. These are only a few of the reasons why correct illumination for your application is so important. The camera's minimum sensitivity is also important in determining the minimum amount of light required in the system. In addition, camera settings such as gain, shutter speed, etc., affect the sensor's sensitivity. There are two methods of illumination which can be used on the described device. Methods of illumination can be divided according to the physical base of illumination source or according to the intensity and the method of illumination of examined volume. During experiments it is very useful to use multiple illumination methods, for example, a combination of light sources listed in the table below. 
Table 1: Illumination methods

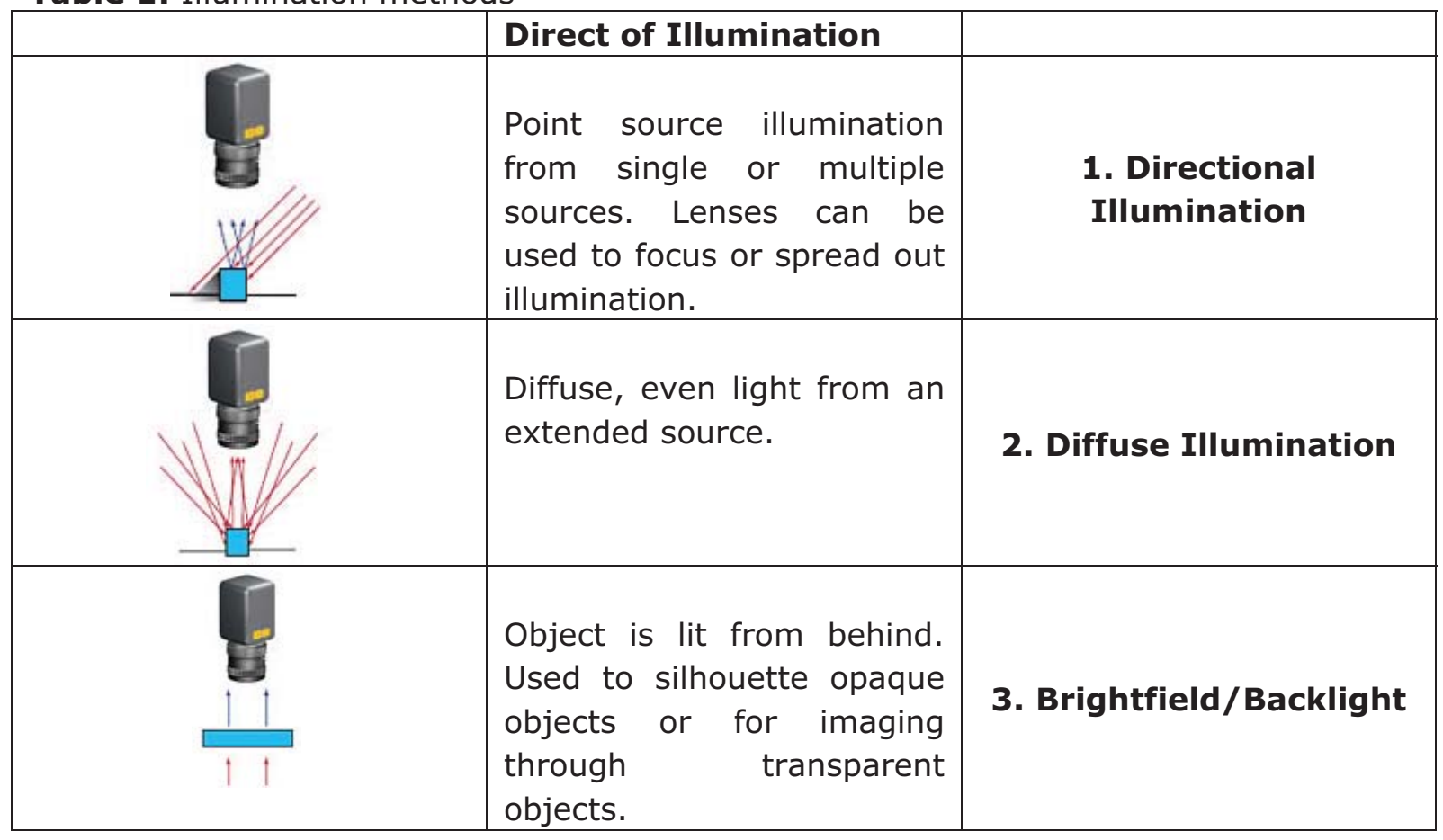

Usage of various light sources for illumination is possible. In discussed application is used: continual microscopic light source (adjustable temperature and wavelength), pulse laser light (green diode module), spark flash lamp (white light mono flash with high energy, or high repetition flashes with low energy).

\section{DAQ AND CONTROL}

The DAQ system is designed for parallel capturing of visual data and hydraulic and thermodynamic parameters of fluid: the pressure of the liquid on input and output collectors, temperature in input and output collectors. For measuring of pressure in selected points of the micro fluidic device two types of sensors are used. For measuring of the static pressure miniature piezoresistive pressure sensors are used with a silicon membrane manufactured by the MEMS technology [3]. For measure of pressure pulsations a piezoelectric pressure sensor is used. The sensor is manufactured with integrated measuring electronics (the measuring amplifier is powered by the power circuit, the measured signal is superposed on the supply voltage, so called ICP technology). Signals are measured by a universal measuring card with sequential A/D conversion with the maximum sample rate $1.2 \mathrm{MHz}$. For the experiment control, recording and analysis of measured data is used measuring software, created in LabView from National Instruments company. In the measuring setup any desired parameter can be set by the block oriented programming environment. Because of very rapid changes in the cavitation phenomenon within the order of $\mu \mathrm{s}$, and in order to have enough light to illuminate the bubble or cloud, the shutter and flash are synchronized using digit output signal from control card. 


\section{Results AND DUSCUSSION}

\section{Vision system setup}

Camera OLYMPUS 7070 WZ

Diffuse Illumination

Continual microscopic light source
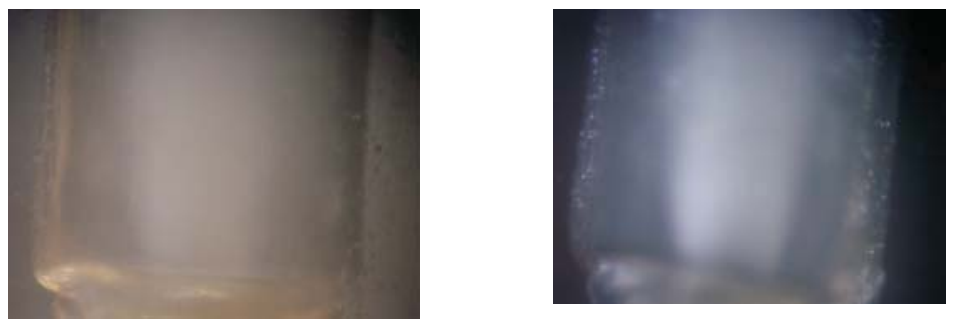

Figure4: Set of experimental video data.

Vision system setup

SONY ExWave

Diffuse Illumination

Continual microscopic light source
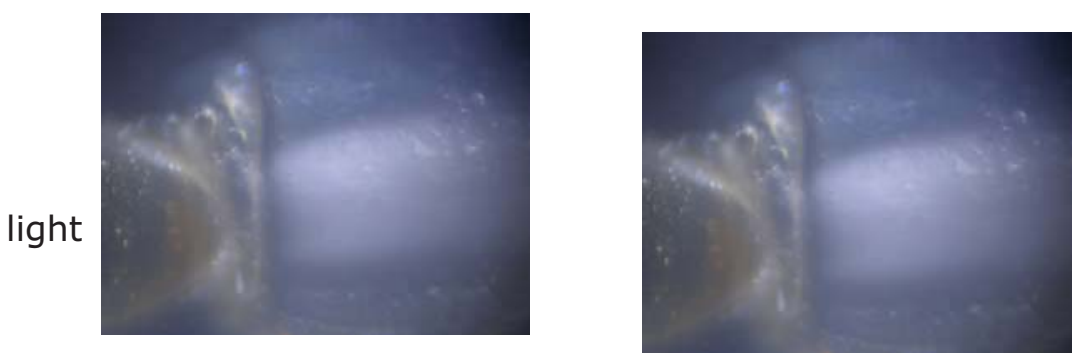

Figure5: Set of experimental video data.

\section{Vision system setup}

Redlake Y3

Diffuse Illumination

Continual microscopic light source
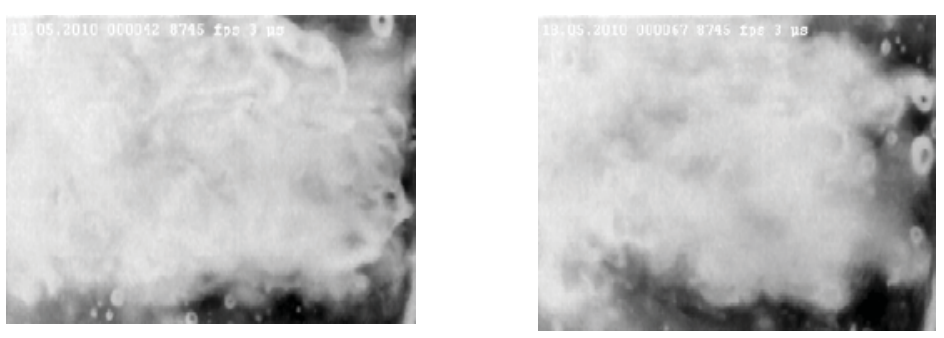

Figure6: Set of experimental video data.

Vision system setup

Redlake Y3

Brightfield/Backlight

Spark flash lamp
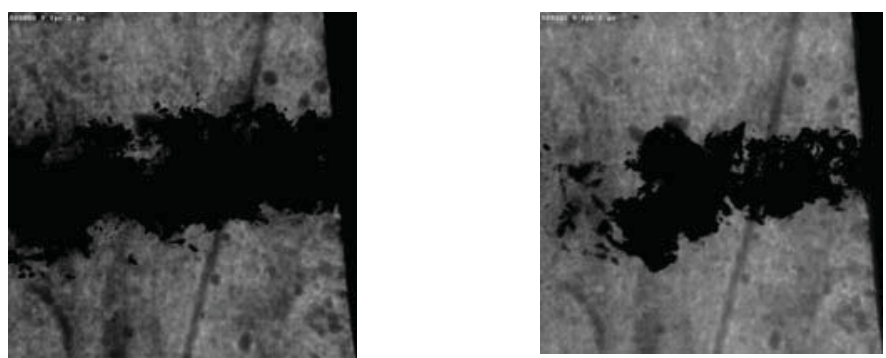

Figure7 Set of experimental video data. 


\section{CONCLUSIONS}

Using of a high speed camera in combination with telecentric objective and pulse white light illumination is very helpful in research of fluid flow in channels of all sizes. In Figure 8 is presented cavitation cloud in the small space inside a microchannel, where a simple flow is assumed, a very complicated flow with number of vortexes can develop as a result of high speeds. The video sequence clearly shows that the cavitation cloud is of a very dynamic structure, pulsing and changing its shape and size in frequency higher than $2 \mathrm{kHz}$. The frequency of capturing is only one of influences which determines the quality of the video. Time of exposition is also very important factor which has a very strong influence on sharpness of the pictures. To "freeze" the motion, this time must be set to value less than $10 \mu \mathrm{s}$ for conditions of our experiment. For exposition short like this, a very intensive illumination is necessary. Illumination is reached by laboratory light source with fibre optics. Through these fibres the light is conducted to the area of the cavitation cloud. It can be testified that the problem of illumination is handled on a very good level and the system of illumination does not need to be changed in the near future.

\section{REFERENCES}

[1] Brennen C.E.: Cavitation and Bubble Dynamics, New York, Oxford, Oxford University Press, 1995

[2] Bahrami M, Yovanovich MM, Culham JR, Pressure drop of fully developed, laminar flow in rough microtubes, PROCEEDINGS OF $3^{\text {rd }}$ INTERNATIONAL CONFERENCE ON MICROCHANNELS AND MINICHANNELS, TORONTO, CANADA, 2005

[3] GAD-EL-HAK, M. The MEMS Handbook. Introduction and Fundamentals. CRC Press Taylor \& Francis Group, 2006.

[4] Hutli E.A, Nedeljkovic, M. Ilic V.: Visualization of Submerged Cavitating Jet: Part One, 16th Australasian Fluid Mechanics Conference Crown Plaza, Gold Coast, Australia 2-7 December 2007, pages 876-880

[5] Chandan M., Peles Y.: Cavitation in flow through a micro-orifice inside a silicon microchannel, PHYSICS OF FLUIDS 17, 013601 (2005), pages 013601-1, 013601-15

[6] NGUYEN, T. N., WERELEY, S. T. Fundamentals and applications of microfluidics. Artech house, 2006.

[7] OLŠIAK, R. Experimentálne zariadenie na výskum prúdenia v kanáloch vel'mi malých rozmerov, Zborník XVII medzinárodnej vedeckej konferencie „Aplikácia experimentálnych a numerických metód v mechanike tekutín a v energetike", Bojnice 2010, ISBN 978-80-554-0189-8

This work was supported by the Scientific Grant Agency VEGA under contract number $1 / 0251 / 11$. 